\title{
$\angle$ Research Square \\ "MORIEF Technique" for Schatzker Type II-III Tibial Plateau Fractures with Tension Blisters: A Retrospective Study of Seven Patients
}

\section{Xin Qi}

920th Hospital of Joint Logistics Support Force

\section{Yong-Qing Xu}

920th Hospital of Joint Logistics Support Force

Hong-Bo Tan

920th Hospital of Joint Logistics Support Force

\section{Shen Xia}

920th Hospital of Joint Logistics Support Force

\section{Xiao-Yan Xu}

920th Hospital of Joint Logistics Support Force

\section{Qian Lv}

920th Hospital of Joint Logistics Support Force

\section{Ze-Yu Zhao}

920th Hospital of Joint Logistics Support Force

Yue-Liang Zhu ( $\square$ zhu123@zju.edu.cn )

2nd Affiliated Hospital,School of Medicine, Zhejiang University.

\section{Research Article}

Keywords: Tibial plateau fracture, Tension blisters, llizarov technique, External fixation

Posted Date: November 22nd, 2021

DOI: https://doi.org/10.21203/rs.3.rs-1078584/v1

License: (c) (1) This work is licensed under a Creative Commons Attribution 4.0 International License. Read Full License 


\section{Abstract}

Background: When tibial plateau fractures are combined with tension blisters, internal fixation surgery must be delayed. However, a prolonged delay may cause complications during fracture treatment. To combat this challenge, we innovatively proposed a minimally open reduction and llizarov external fixation (MORIEF) technology. Therefore, the present study aimed to explore the treatment, safety, and efficacy of the MORIEF technique in Schatzker type II-III tibial plateau fractures with tension blisters.

Methods: We retrospectively analyzed data of seven patients with Schatzker type II-III tibial plateau fractures with tension blisters treated at our hospital from September 2013 to March 2017. All patients (five males, two females; mean age: $38.1 \pm 8.2$ [range: $28-50$ ] years) underwent the MORIEF technique. The Knee Society Score (KSS) and assessment of bone status according to the Association for the Study and Application of the Method of Ilizarov (ASAMI) scoring system were used to evaluate the knee joint function and bone healing condition.

Results: The operative time was $108.6 \pm 14.6$ (range: $90-135)$ min, blood loss was $104.3 \pm 50.4$ (range: $50-$ 200) $\mathrm{ml}$, time from operation to discharge was $2.6 \pm 0.7$ (range: $2-4$ ) days, and the follow-up period was $20.6 \pm 1.8$ (range: $18-22$ ) weeks. The fractures healed in all patients at 11.9 \pm 1.1 (range: 10-14) weeks. Except for one case of needle tract infection, no other complications occurred. At the last follow-up, the KSS presented a clinical score of 80-95 (mean: 86.4 \pm 4.4 ) points and a functional score of 85-95 (mean: 87.9 \pm 3.6 ) points. According to ASAMI, four cases were classified as excellent, two as good, one as fair, and none as poor.

Conclusions: The use of the MORIEF technology for the treatment of Schatzker type II-III tibial plateau fractures with tension blisters showed that the resulting incidence of infection is low, weight-bearing and walking can be resumed immediately, hospitalization time is shortened, economic burden of patients is reduced, and fracture healing and joint function recovery are not affected postoperatively. Therefore, this technique was shown to be a relatively safe and effective treatment method for the condition.

\section{Background}

Tibial plateau fractures are common intra-articular fractures, accounting for approximately $1.2 \%$ of all body fractures [1]. With the diversification of injury factors, their incidence has been gradually increasing and their occurrence is often complicated by severe and extensive soft tissue injuries [2-4], usually leading to a poor prognosis. This study focused on Schatzker II-III tibial plateau fractures with tension vesicles, which have some particularities. The particularity of skin tension blisters is that the skin and soft tissues are seriously damaged and easily complicated by osteofascial syndrome, which aggravates the condition. Closed fractures may develop into open fractures, potentially leading to life-threatening concurrent infections and increasing the difficulty of treatment and risk of amputation.

Traditional surgical methods, including internal fixation techniques such as open reduction, minimally invasive percutaneous plate internal fixation (MIPPO), and minimally invasive surgery, as well as external 
fixation techniques (e.g., hybrid external fixation) require a long time (7-10 days or even 2 weeks) before the operation aimed at reducing the swelling is performed. The risk of complications such as infection, nonunion, and malunion after surgery is high, with an incidence of approximately $10 \%$. To date, minimally invasive reduction and fixation are the main trends in the treatment of fractures, and, in recent years, many scholars have studied the llizarov technique as a novel treatment [5-8], especially for fractures presenting soft tissue injuries. Due to the aforementioned challenges in China and globally, the author of this study proposed the minimally open reduction and llizarov external fixation (MORIEF) technology as a potential treatment option, which has advantages with respect to the shortened waiting time for soft tissue edema and skin tonic water blisters, reduced number of days in the hospital, immediate weightbearing and return to life after surgery, avoidance of re-hospitalization to remove the internal fixation, low wound infection rate, and absent need for flaps. This study aimed to explore the methods, safety, and clinical efficacy of this technique in the treatment of Schatzker type II-III tibial plateau fractures with tension blisters and report the resulting minimum trauma and maximum functional recovery [9].

\section{Material And Methods}

\subsection{Inclusion and exclusion criteria}

The inclusion criteria were as follows: (1) Patients over 18 years of age with mature bones and no osteoporosis, (2) those diagnosed with closed tibial plateau fractures confirmed by imaging examination, (3) those with skin tension blisters who have not developed osteofascial syndrome, and (4) those who could complete at least an 18-week follow-up.

The exclusion criteria were as follows: (1) Patients with osteoporosis, (2) those with open tibial plateau fractures, (3) those with multiple fractures of the tibia (middle or distal), (4) those with nerve and blood vessel damage, (5) those with knee joint meniscus and ligament injuries, (6) those with joint deformities caused by knee osteoarthritis, traumatic arthritis, and rheumatic joints, (7) those with pathological fracture, and (8) those with underlying diseases such as coronary heart disease, hypertension, and diabetes.

\subsection{General data}

Seven patients were included in this study. There were five males and two females with an average age of $38.1 \pm 8.2$ (range: $28-50$ ) years. The injury factors included three cases of traffic accident injuries, one case of falling from a height, one case of contusion, and two cases of heavy object injury. Four patients had right fractures and three patients had left fractures. There were four cases of type II and three cases of type III Schatzker tibial plateau fractures. The time from injury to admission was $1-10 \mathrm{~h}$, with an average of $5.1 \pm 2.9 \mathrm{~h}$. The time from admission to operation was $1-3$ days, with an average of $1.6 \pm 0.7$ days. There were six cases of simple tibial plateau fractures and one case of combined fractures of the proximal fibula on the affected side. All seven cases were complicated by tension blisters. After admission, the affected limb was raised to reduce swelling, blisters were treated to prevent infection, and no compartment syndrome occurred before and after surgery. This study was performed following the 
principles of the Helsinki Declaration. All patients signed a surgical consent form before surgery. This research has been approved by the institutional review boards of the authors' affiliated institutions(920th Hospital of Joint Logistics Support Force).

\subsection{MORIEF Technology}

Regarding Schatzker type II-III tibial plateau fractures with tension blisters, the traditional treatment method involves waiting for the soft tissue and skin blisters to fully improve and the subsequent performance of open reduction and plate internal fixation, however, the incision of the traditional treatment method is larger and the risk of infection is higher. The technical incision made in the present study is smaller and uses Kirschner wire to fix the distal and proximal ends of the fracture, and, more importantly, gives full play to the reduction and compression of the olive needle.

Under general anesthesia, the patients were placed in the supine position, the affected limb was disinfected, and surgical shop towels were placed in the surgical area. Due to the presence of tension blisters, after minimally invasive open reduction, llizarov external fixation was performed in the absence of internal fixation; this technique is the core of the MORIEF technology.

For the minimally invasive open reduction technique, an incision of approximately $5-8 \mathrm{~cm}$ in length at the tibial plateau fracture was made, gradually revealing the fractured ends and protecting soft tissue and periosteum blood supply to avoid further damage. Manipulative traction with Kirschner wires was performed to pry and reset the fractured end and restore the collapsed articular surface. When necessary, bone grafting was performed, which was required for three cases of autologous iliac bone grafting in this sample. Approximately 1-2 Kirschner wires were used for temporary fixation after anatomical reduction. When the $\mathrm{C}$-arm $\mathrm{X}$-ray machine fluoroscopy indicated good reduction, the surgical port was washed and disinfected, and the entire layer was sutured with surgical suture.

For the llizarov external fixation technique, four tibial rings were prepared, including one "C" ring near the fractured end and three full rings at the far side. The rings were connected as a whole with 3-4 threaded rods of appropriate length. The affected limb was placed in the center of the ring frame, and the assistant maintained the ring frame position. The surgeon cross-fixed 1-3 olive needles at the broken end of the fracture. Two $2.0 \mathrm{~mm}$ or $2.5 \mathrm{~mm}$ full needles were drilled into the four circular frame planes at the distal and proximal ends of the tibial plateau fracture from the outside to the inside, ensuring to avoid drilling into the joints. One $3.0 \mathrm{~mm}$ and one $4.0 \mathrm{~mm}$ half needles were drilled into the distal and proximal ends of the fracture respectively. The stability of all fixed pins was checked using special accessories to securely fasten the fixing pin to the ring-shaped outer fixing frame. A distractor was used to pull the olive needle to reduce the fractured end, fix it with compression, and then tighten the nut to fix it. Subsequently, the C-arm X-ray machine was used to re-examine the functional position of the knee joint; once it was confirmed that the fracture reduction was sufficient and the position of the external fixation frame was appropriate, the half needle was cut, and the olive and full needles were bent. The needle path was disinfected and the surgical port was wrapped with a sterile dressing.

\subsection{Postoperative management and followup}


Symptomatic treatments, such as anti-infection with antibiotics and analgesia, were administered after surgery. In this study, three patients with thrombosis of the lower limbs underwent anticoagulation and thrombolytic therapy on the first postoperative day. The patients were instructed to continually raise the affected limb to reduce swelling. Routine disinfection and dressing change were performed, and the incision and needle path were kept clean and dry to avoid infection.

Functional exercise of adjacent joints was performed on the first postoperative day and review radiographs were then performed in the hospital. On the second day after the operation, the patients without bone grafts were fully weight-bearing and those with bone grafts were partially weight-bearing, gradually achieving full weight-bearing within 2 weeks. After 10-14 weeks, the external fixation device was gradually removed according to the fracture healing condition. To ensure safety, a step-by-step dynamic method combined with weight-bearing pain response was used [9]; our method involved loosening the nut first and then removing the fixed needle. After the radiograph showed that the bone was healed, the nut was loosened, and the patient was instructed to continue walking with weight. In addition, 1-2 fixed needles were removed from each unit every 2 weeks until they were all removed. The removal is completed within 14-16 weeks after the operation and is performed in the outpatient or dressing room without anesthesia and re-admission. At 18-22 weeks postoperatively, the Knee Society Score (KSS) [10] and assessment of bone status by the Association for the Study and Application of the Method of llizarov (ASAMI) scoring system [4] were used to evaluate knee joint function and bone healing at the last follow-up.

\section{Results}

The operative time was $108.6 \pm 14.6$ (range: $90-135$ ) min and the blood loss was $104.3 \pm 50.4$ (range: $50-$ 200) $\mathrm{mL}$. The time from operation to discharge was $2-4$ days, with an average of $2.6 \pm 0.7$ days. All patients were followed up after surgery. The follow-up period was $20.6 \pm 1.8$ (range: 18-22) weeks. The fractures healed in all patients, and the healing time was 11.9 1.1 (range: 10-14) weeks. One patient showed signs of needle tract infection, which was effectively controlled within 2 weeks of using oral antibiotics combined with strict needle cleaning, sterile dressing changes, and other nursing measures. During the follow-up period, there were no complications such as fracture displacement, deep infection of the incision site, osteomyelitis, delayed bone union or nonunion, and joint stiffness. At the last follow-up, the knee joint KSS clinical score was $40-45$ for pain (average: $42.1 \pm 2.5$ points), $20-25$ (average: 22.2 \pm 2.5 ) for range of motion, and 20-25 (average: $22.1 \pm 2.5$ ) for stability, with a total score of 80-95 (average: $86.4 \pm 4.4$ ). Functional scores were $45-50$ (average: $46.4 \pm 2.3$ ) for walking ability and $40-45$ (average: $41.5 \pm 2.3$ points) for stairs ability, with a total score of $85-95$ (average: $87.9 \pm 3.6$ ). According to ASAMI, four cases were excellent, two cases were good, one case was fair, and no case was poor. Therefore, the excellent and good rates accounted for $85.7 \%$ of cases (Table 1) (Figures 1 and 2 for examples of typical cases). 
Table 1

Assessment of bone status by the ASAMI scoring system

\begin{tabular}{|c|c|c|c|}
\hline Standard & $\begin{array}{l}\text { Bone } \\
\text { results }\end{array}$ & $\mathbf{N}$ & $\%$ \\
\hline Union, no infection, deformity $<7^{\circ}$, limb length discrepancy $<2.5 \mathrm{~cm}$ & Excellent & 4 & 57.1 \\
\hline $\begin{array}{l}\text { Union + any two of the following: no infection, deformity }<7^{\circ} \text {, limb length } \\
\text { discrepancy }<2.5 \mathrm{~cm}\end{array}$ & Good & 2 & 28.6 \\
\hline $\begin{array}{l}\text { Union }+ \text { only one of the following: no infection, deformity }<7^{\circ} \text {, limb length } \\
\text { discrepancy }<2.5 \mathrm{~cm}\end{array}$ & Fair & 1 & 14.3 \\
\hline $\begin{array}{l}\text { Nonunion/refracture/union }+ \text { infection }+ \text { deformity }>7^{\circ}+\text { limb length } \\
\text { discrepancy }>2.5 \mathrm{~cm}\end{array}$ & Poor & 0 & 0 \\
\hline
\end{tabular}

ASAMI: Association for the Study and Application of the Method of Ilizarov

\section{Discussion}

It is usually difficult to achieve good results for tibial plateau fractures, mainly because of the various severe complications with potentially destructive consequences [11]. Therefore, a feasible choice of surgical method is important to investigate for the treatment of these conditions. This study specifically aimed to explore an innovative treatment plan for Schatzker type II-III tibial plateau fractures with tension blisters, showing promising results. This study is a retrospective study and does not account for gender differences.

\subsection{Traditional treatment methods}

Currently, open reduction and internal fixation (ORIF) is the mainstream method. The advantage of this technique is the ability to achieve the visualization of the fracture and articular surface reduction [6] and perform absolute anatomical reduction and rigid fixation. Nevertheless, the main disadvantage is that the operation causes further damage to the soft tissue and bone structure, which increases the risk of various complications, as well as presenting a very long surgical waiting time due to the soft tissue swelling and not allowing for early weight-bearing. The advent of minimally invasive open reduction and internal fixation (MORIF), such as MIPPO, has optimized the effect of stage fixation and reduced surgical trauma, but the aforementioned problems cannot be completely avoided $[12,13]$.

Hybrid combined external fixation is also an option, with advantages that include a simple operation, minimal surgical trauma, and protection of soft tissues; however, unilateral eccentric fixation, the reset effect, and fixation stability are not effective and can only be used for temporary fixation.

Although we do not deny the significance of traditional methods such as ORIF and hybrid external fixation, this study demonstrated the use of an improved treatment option for the treatment of Schatzker type II-III tibial plateau fractures with tension blisters, as discussed below. 


\subsection{Primary experience with the MORIEF technology}

There is no clear gold standard technique for the surgical treatment of tibial plateau fractures [14]. This study implemented the MORIEF technology, which uses the llizarov external fixation technology as the core technique. The excellent orthopedic function of the llizarov technique has long been recognized by the medical community; however, it has been ignored for the treatment of fractures. For the treatment of tibial plateau fractures, some scholars have proposed the surgical method of llizarov external fixation combined with ORIF, which is considered the gold standard for minimizing complications [15]. With the continuous updating of technology, the combination of minimally invasive techniques for joint reduction, such as prying and arthroscopic reductions, with externally stabilized llizarov external fixation has become an effective treatment option and has gradually developed into the concept of llizarov external fixation surgery with or without minimal internal fixation $[2,14]$. The MORIEF technology used in this study is a new option to further minimize surgical damage and maximize repair and reconstruction.

The first novel feature of the proposed technology is its extended minimally invasive nature. First, when the fixed needle is drilled in, it is wrapped with sterile gauze soaked in ice saline to reduce thermal damage. Second, the limited surgical port and prying reduction minimize the extensive dissection of soft tissue and periosteum. Finally, no plate is implanted to avoid additional loss of bone structure. Whether this technique can be classified as minimally invasive should be based on the presence or absence of the incision or size of the incision as the only criterion.

The second new feature is the concept of flexible needle threading and circular structure. First, the olive needle is drilled through the skin perpendicular to the longitudinal axis of the tibia or the fracture line. The olive pit near the broken end is placed on the convex side, whereas that far from the broken end is placed on the concave side. The distractor appropriately pulls the tail of the needle for compression and is then fixed on the ring frame, instead of screws, to maintain compression and fixation; the ring frame can then be used for necessary adjustments after the operation. Studies have shown that the use of olive needles does not affect the outcome of the treatment of tibial plateau fractures [2]. Indeed, the use of olive needles at the fracture ends improves the recognized shortcomings of llizarov external fixation, which is the "inability to fix the fracture fragments well, and it is difficult to obtain a better reduction effect" [3]. Second, the threaded rod and the ring frame are connected as a whole, and the fixation pin is firmly fixed across the fracture line, replacing the steel plate, following the principle of central fixation and neutralizing stress, which avoids stress shielding and osteoporosis or even fracture recurrence.

\subsection{The advantages of the MORIEF technology and situations that require attention}

Tibial plateau fractures are often accompanied by extensive and severe soft tissue damage, and the degree of this damage is an important indicator of functional prognosis [16]. Ilizarov technology, due to its tension-stress principle [17], presents more benefits than those of ORIF, including soft tissue protection, early weight-bearing, and adjustment and restoration of normal gait [18], as well as having a high safety 
profile. As a method for treating Schatzker type II-III tibial plateau fractures with tension blisters, it is worthy of clinical application.

The advantages of MORIEF technology include: (1) The minimization of soft tissue damage, including the absence of the need for plate implantation during the operation, no further expansion, limited volumetric occupation of subcutaneous tissue, and ease of closing the surgical wound; (2) the surgery can be performed early, as there is no need to wait a long time for soft tissue swelling and tension blisters to subside, and the preoperative preparation time is minimized; (3) the risk of complicated osteofascial syndrome is minimized, as we believe that the percutaneous needle also serves as a decompression method; (4) the fixation is firm and flexible, as the tension of the ring frame and the fixed pin can hold the cancellous bone, prevent the fracture from collapsing again, and concurrently form a bridge, and the weight-bearing force can bypass the fractured area [16]; (5) the early achievement of postoperative weight-bearing and joint function exercises eases the process for both doctors and patients, it is not only a worry-free for the patients, but also for the doctors; (6) the patient is able to walk and resume life immediately after the operation, effectively shortening the hospitalization time and reducing the economic burden of the patient; (7) according to the fracture healing condition, the ring frame device can be adjusted when necessary to reduce the gap between fracture ends and promote fracture healing; (8) there is no need for hospital re-admission for implant removal surgery, as the implant can be easily removed in the outpatient clinic or dressing room, avoiding any anesthesia-derived risks and further reducing the patient's economic burden.

Nevertheless, a few points of caution need to be noted about the MORIEF technology: (1) Clinicians should be careful to avoid important surrounding blood vessels, nerves, and tendons when piercing the needle during the operation; (2) if there is a serious infection in the surgical port, the external fixation configuration needs to be adjusted or even removed in advance, the fracture may be displaced, and the treatment will fail; (3) postoperative needle-tract care requires higher standards than internal fixation does, as there is a significant incidence of needle tract infection, which increases the risk of deep infection; (4) some patients complain of inconvenience caused by external fixation, but this does not affect their normal life; (5) the frequency of follow-up is high, and some patients have poor compliance and are easily lost to follow-up.

In summary, MORIEF technology for the treatment of Schatzker type II-III tibial plateau fractures with tension blisters (during the dangerous period of acute soft tissue edema) was shown not to require prolonged surgical waiting times (due to swelling) to minimize the preoperative preparation time and avoid further damage to the soft tissue during the operation; the wound is in fact easy to close. After the operation, the incidence of infection is low, weight-bearing and walking can be resumed immediately, hospitalization time is shortened, the economic burden of patients is reduced, and fracture healing and joint function recovery are not affected. Therefore, the findings of our study suggest that this technology is a relatively safe and effective treatment method for Schatzker type II- III tibial plateau fractures with tension blisters. 


\section{Abbreviations}

ASAMI

Association for the Study and Application of the Method of llizarov

KSS

Knee Society Scoring System

MIPPO

Minimally invasive percutaneous plate internal fixation

MORIEF

Minimally open reduction and llizarov external fixation

MORIF

Minimally invasive open reduction and internal fixation

ORIF

Open reduction and internal fixation

\section{Declarations}

Ethics approval and consent to participate

This study has been approved by the Ethics Committee of Kunming General Hospital of Chengdu Military Command,Approval number:(()2018076(920th Hospital of Joint Logistics Support Force).

This study was performed following the principles of the Helsinki Declaration.

This study obtained written consent from all participants.All patients signed a surgical consent form before surgery.

Consent for publication

The data,including any individual details and images of individuals involved in this study have been approved by the patients.

Availability of data and materials

The datasets used and/or analysed during the current study are available from the corresponding author on reasonable request.

Competing interests

The authors declare that they have no competing interests.

Funding 
Foundation item: The key project of construction of clinical center of Yunnan Provincial Health Commission (Project Number: ZX20191001).

This funding body provides publication fee support for this manuscript.The funding bodies played no role in the design of the study and collection, analysis, and interpretation of data and in writing the manuscript.

\section{Author's contributions}

YQX,HBT,SX,XYX,QL and ZYZ analyzed and interpreted the patient data regarding the Schatzker type II-III tibial plateau fractures with tension blisters. XQ and YLZ performed the collection of follow-up data , and were major contributors in writing the manuscript. All authors read and approved the final manuscript.

\section{Acknowledgments}

The authors gratefully acknowledge the following individuals from 920th Hospital of Joint Logistics Support Force and 2nd Affiliated Hospital of Zhejiang University for their collaboration and assistance with this study: Shao-Quan Pu, Sun-Wen Pan, Zhi-Jun Cai, Xing-Jian Qian, Xing-Long Pan, and Han-Fen Chen. In addition, we would like to thank Editage (www.editage.cn) for their English language editing services.

Authors have been obtained permission to acknowledge from all those mentioned in the acknowledgements section.

\section{References}

[1] Lee MH, Hsu CJ, Lin KC, Renn JH. Comparison of outcome of unilateral locking plate and dual plating in the treatment of bicondylar tibial plateau fractures. J Orthop Surg Res. 2014;9:62.

[2] Subramanyam KN, Tammanaiah M, Mundargi AV, Bhoskar RN, Reddy PS. Outcome of complex tibial plateau fractures with llizarov external fixation with or without minimal internal fixation. Chin $\mathrm{J}$ Traumatol. 2019;22:166-171.

[3] Thiagarajah S, Hancock GE, Mills EJ, McGregor-Riley JC, Royston SL, Dennison MG. Malreduction of tibial articular width in bicondylar tibial plateau fractures treated with circular external fixation is associated with post-traumatic osteoarthritis. J Orthop. 2019;16:91-96.

[4] Lalić I, Daraboš N, Stanković M, Gojković Z, Obradović M, Marić D. Treatment of complex tibial plateau fractures using Ilizarov technique. Acta Clin Croat. 2014;53:437-48.

[5] Debnath UK, Jha DK, Pujari PK. Results of ring (Ilizarov) fixator in high energy Schatzker type VI fractures of proximal tibia. J Clin Orthop Trauma. 2018;9:186-191. 
[6] Tahir M, Kumar S, Shaikh SA, Jamali AR. Comparison of postoperative outcomes between open reduction and internal fixation and llizarov for Schatzker type V and type VI fractures. Cureus. 2019;11:e4902.

[7] Keightley AJ, Nawaz SZ, Jacob JT, Unnithan A, Elliott DS, Khaleel A. llizarov management of Schatzker IV to VI fractures of the tibial plateau: 105 fractures at a mean follow-up of 7.8 years. Bone Joint $\mathrm{J}$. 2015;97-B:1693-1697.

[8] Ganadhiepan G, Miramini S, Patel M, Mendis P, Zhang L. Bone fracture healing under Ilizarov fixator: Influence of fixator configuration, fracture geometry, and loading. Int J Numer Method Biomed Eng. 2019;35:e3199.

[9] Jabbar Y, Jeyaseelan L, Khaleel A. Staged complete dynamisation of the llizarov fixator: The Chertsey experience. Eur J Orthop Surg Traumatol 2011;21:521-526.

[10] Insall JN, Dorr LD, Scott RD, Scott WN. Rationale of the Knee Society clinical rating system. Clin Orthop Relat Res. 1989;248:13-14.

[11] Farooq U, Javed S, Ahmad I, Aziz A. Functional outcome of complex tibial plateau fractures managed with closed ilizarov. J Pak Med Assoc. 2014;64 Suppl 12:104-107.

[12] Ozkaya U, Parmaksizoglu AS. Dual locked plating of unstable bicondylar tibial plateau fractures. Injury. 2015;46 Suppl 2:9-13.

[13] Ruffolo MR, Gettys FK, Montijo HE, Seymour RB, Karunakar MA. Complications of high-energy bicondylar tibial plateau fractures treated with dual plating through 2 incisions. J Orthop Trauma. 2015;29:85-90.

[14] Ali AM. Outcomes of open bicondylar tibial plateau fractures treated with Ilizarov external fixator with or without minimal internal fixation. Eur J Orthop Surg Traumatol. 2013;23:349-355.

[15] Bove F, Sala F, Capitani P, Thabet AM, Scita V, Spagnolo R. Treatment of fractures of the tibial plateau (Schatzker VI) with external fixators versus plate osteosynthesis. Injury. 2018;49 Suppl 3:12-18.

[16] Raza A, Kumar S, Kumar D, Qadir A, Muzzammil M, Lakho MT. Complex Tibial Plateau Fractures: Primary Fixation Using the llizarov External Fixator. A Two-year Study at Civil Hospital Karachi, Pakistan. Cureus. 2019;11:e5375.

[17] Frost HM. Wolff's Law and bone's structural adaptations to mechanical usage: an overview for clinicians. Angle Orthod. 1994;64:175-188.

[18] Iliopoulos E, Agarwal S, Khaleel A. Walking impairments after severe tibia plateau fractures. A gait pattern analysis. J Orthop Sci. 2020;25:276-278. 


\section{Figures}
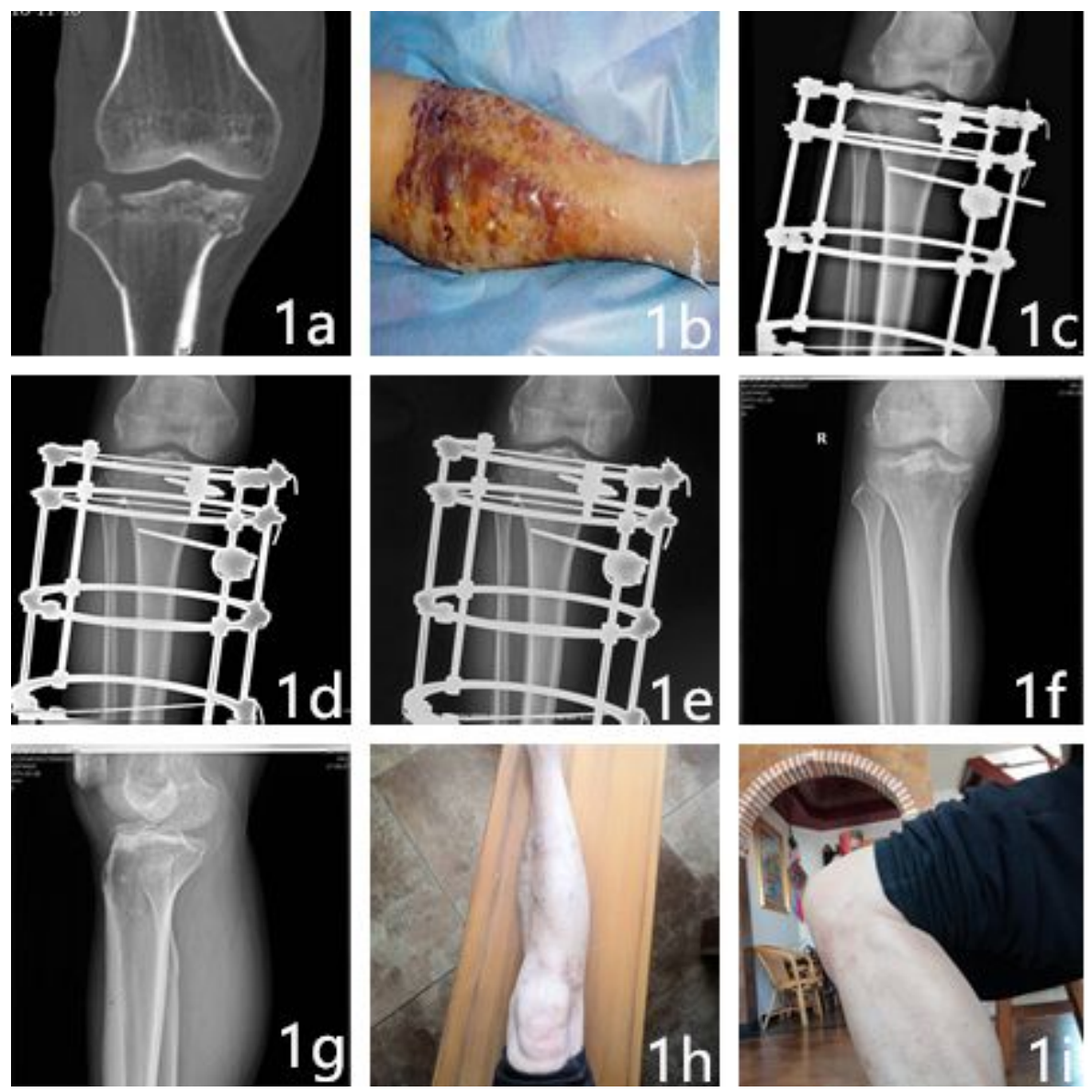

\section{Figure 1}

A 43-year-old man with a right tibial plateau fracture with skin tension blisters and large bruises: a) CT plain scan image before the operation; b) Preoperative appearance of tension blisters; c) Reexamination of radiographs on the first postoperative day, indicating a good reduction of the fracture end with the external fixation firmly in place; d) Reexamination of radiographs at 8 weeks after the operation, indicating that the callus has begun to grow but the bone has not healed completely; e) Reexamination of radiographs at 12 weeks after the operation, showing that the fracture line is blurred, indicating bone healing and that the external fixation device can be removed; $f, g, h, i)$ Reexamination of radiographs at 22 weeks postoperatively shows complete bone healing and good knee joint function. 

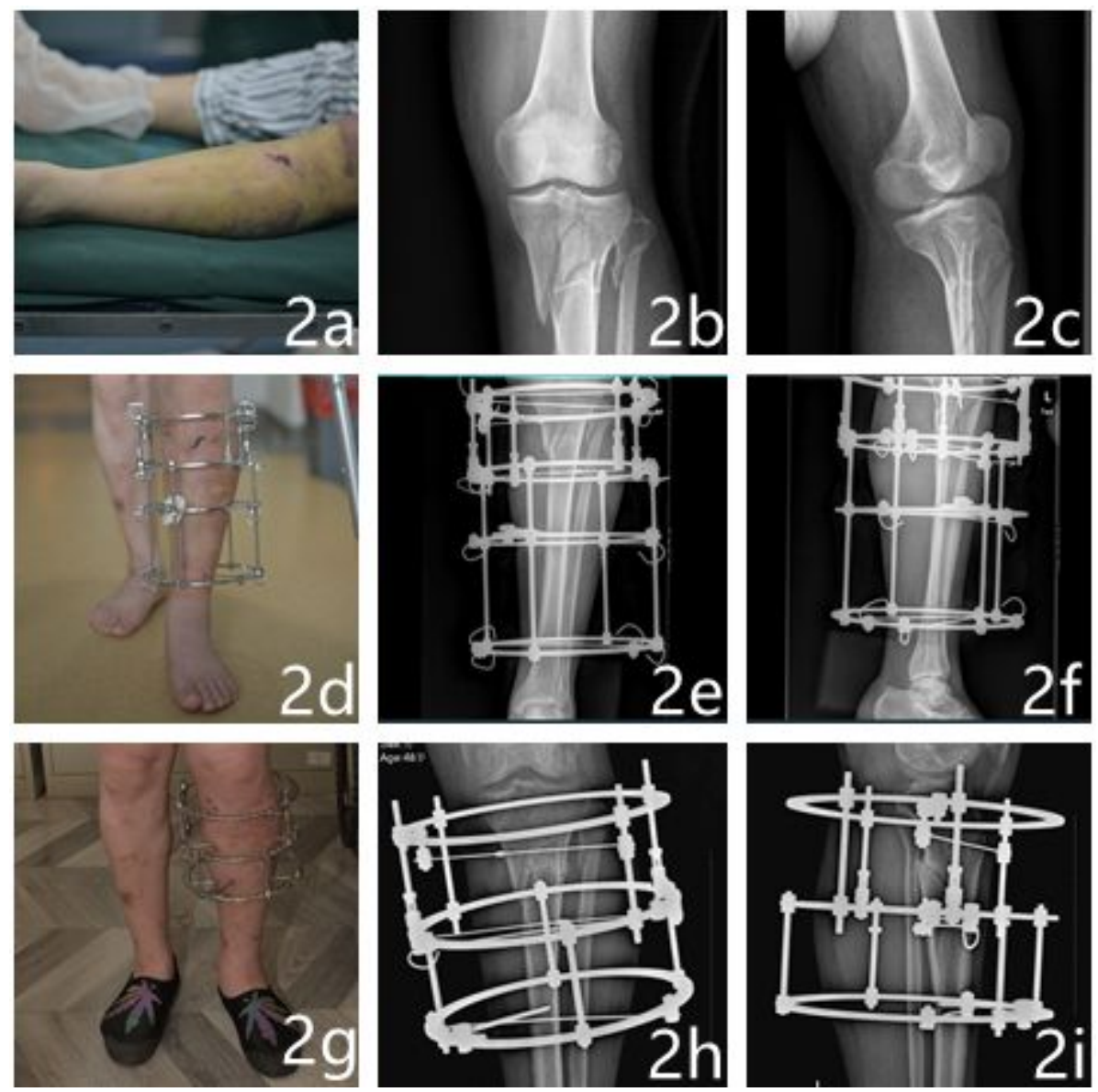

Figure 2

A 48-year-old man with left tibial plateau fracture with skin tension blisters and large bruises: a) Preoperative appearance of tension blisters; $b, c$ ) Radiographs before surgery; d) Postoperative appearance; e,f) Reexamination of radiographs on the first postoperative day, indicating a good reduction of the fracture end with the external fixation firmly in place; g) Appearance at 12 weeks postoperatively, when the distal ring external fixation unit is removed because the fracture begins to heal; $h, i)$ Reexamination of radiographs at 18 weeks after the operation, indicating that the fracture has healed well. 